\title{
Dissociation of Catecholamine and Corticosterone Responses to Different Types of Stress in Rats
}

\author{
Ayako SUDO* and Keiichi MIKI \\ National Institute of Industrial Health, \\ 21-1, Nagao 6-chome, Tama-ku, Kawasaki 214, Japan
}

(Received May 7, 1993 and in revised form June 30, 1993)

\begin{abstract}
To examine hormonal response patterns to various stresses, urinary excretion of catecholamines and corticosterone was measured in spontaneously hypertensive rats (SHR) and Wistar-Kyoto normotensive rats (WKY) under the folowing conditions: immobilization, restiriction to a small space, introduction of new rats, and noise exposure. In WKY rats, immobilization caused a marked increase in urinary corticosterone, adrenaline and noradrenaline and a decrease in dopamine excretion. Restriction to a small space induced a less pronounced but still obvious increase in adrenaline and corticosterone and a decrease in dopamine. When other rats were introduced into the animal room, the senior rats showed an increase in adrenaline alone, while noise exposure produced an increase in corticosterone alone. These findings suggest that while severe stress, such as immobilization, causes marked changes in all 4 hormones, relatively mild stress produces changes in one to three, not all, hormones, and that catecholamine and corticosteroid responses are dissociated in some forms of the mild stress. It is considered that neuroendocrine responses to stress vary according to both the type of stress and its intensity.

In SHR rats, the hormone response to severe stress was greater than in WKY rats, and the response was somewhat less than in WKY rats when exposed to mild stress. These findings do not imply that stress-induced hyperactivity of sympathetic adrenomedullary system participates in the development of hypertension in SHR rats.
\end{abstract}

Key words: Catecholamines - Adrenaline - Noradrenaline-Dopamine-CorticosteroneStress-WKY-SHR-Urine Analysis

\section{INTRODUCTION}

Experimental studies on stress in animals have mostly been performed using immobilization or footshock. Such severe stress undoubtedly produces striking changes in the activity of the sympathetic adrenomedullary system and the pituitary-adrenocortical axis ${ }^{1,2)}$. Several workers ${ }^{3,4,5)}$ have reported that less severe stress, such as handling or exposure to a novel environment, also induces

* To whom all correspondence should be addressed 
changes in plasma levels of catecholamines and corticosteroids in animals.

Plasma concentrations of catecholamines and corticosterone have been measured in many of the studies performed on rodents to assess the activity of these two neuroendocrine systems. Blood sampling, however, involves animal restraint and venipuncture which may provoke psychological and physiological disturbances. Indwelling catheters can be employed to avoid such undesirable effects ${ }^{6,7)}$, but the results tend to be influenced by the skillfulness of surgical operation and the quality of animal care. Moreover, the effect of an indwelling needle and blood loss on the circulatory system cannot be completely excluded.

Another way to investigate hormonal changes is urine analysis. Since urine collection usually requires no detrimental treatment, analyzing urine rather than blood would seem to provide information of practical value regarding the changes induced, especially by mild stress. We have developed sensitive methods of HPLC and post-column fluorometry to analyze urinary corticosteroids ${ }^{8)}$ and catecholamines $^{9)}$.

The aim of the present study was to examine urinary excretion of catecholamines and corticosterone in rats exposed to various types of stress, and to compare the patterns of changes induced by mild stress with those induced by severe stress. To achieve this aim, rats were exposed to several kinds of stress and urinary excretion of corticosterone, adrenaline, noradrenaline and dopamine was measured. The data were obtained from spontaneously hypertensive rats (SHR) and Wistar-Kyoto normotensive rats (WKY) to elucidate whether SHR rats are more responsive to mild stress, as has been previously reported ${ }^{10)}$ in the case of severe stress.

\section{Methods}

\section{Animals and Housing}

The male SHR and male WKY rats used in this study were obtained from Charles-River Japan at the age of 4 weeks. They were housed individually in 20 $\times 20 \times 20 \mathrm{~cm}$ (width $\times$ depth $\times$ height) metabolic cages prepared for urine collection, and were allowed to adapt to the cage for 10 days prior to the experiment. Temperature and humidity were controlled at $25 \pm 1{ }^{\circ} \mathrm{C}$ and $55 \pm 5 \%$, repectively. Food and water were given ad libitum. The light period was fixed at a 12-12 hour reversed cycle (lights on: $21: 00^{-9}: 00$ in stress experiment 1 and $20: 30^{-}$ $8: 30$ in stress experiment 2). The experiments were carried out when the rats were between 6 and 8 weeks old.

\section{Stress Experiment 1}

The stress used in this experiment were as follows: 1 , a white, $19 \times 16 \times 19 \mathrm{~cm}$ plastic box was placed in each metabolic cage, greatly reducing the free space in the cage ('RESTRICTED SPACE' CONDITION), but the rats were able to 
move in the remaining space because they were not immobilized; 2,3 other SHR rats were introduced into the experiment room and maintained in the same manner as described above ('NEW RAT IN ROOM' CONDITION); 3, intermittent $75 \mathrm{~dB}(\mathrm{C}), 100 \mathrm{~dB}(\mathrm{C})$ or $85 \mathrm{~dB}(\mathrm{C})$ white noise $(300-8000 \mathrm{~Hz}, 2-$ min duration, fixed interstimulus intervals of $2 \mathrm{~min}$ ) for 4 hours; 4 , immobilization by wire mesh for $20 \mathrm{~min}$ or for 2 hours. Stress exposure began at 10:00. Seven SHR rats and 8 WKY rats were exposed to these 7 types of stress in that order, at intervals of 3 days. The mean body weights of the SHR and WKY rats immediately before the 'RESCTRICTED SPACE' experiment were $162 \pm 3 \mathrm{~g}$ (mean $\pm \mathrm{SEM}$ ) and $162 \pm 4 \mathrm{~g}$, respectively. Every experimental day, two 4-hour urine samples, from the start of stress exposure to $14: 00$ (stress period) and from $14: 00$ to $18: 00$ (recovery period), were collected from each rat in glass bottles containing $1 \mathrm{ml}$ of $1 \mathrm{~N} \mathrm{H}_{2} \mathrm{SO}_{4}$. Urine collection was performed in the same manner on the day immediately before the day of stress exposure (control day), with the exception of the 'NEW RAT IN ROOM' condition.

The noise level was measured with a sound meter (Sound Meter, SM-6, Onsoku, Tokyo) in front of the rat cage, and the noise level for individual cages ranged within $\pm 2 \mathrm{~dB}(\mathrm{C})$. The background noise level during the experiment was 55-60 dB(C).

Three days after the conclusion of the experiment, the systolic blood pressure and heart rate of the rats were measured by the tail-cuff method (MK-1000, Muromachi). Systolic blood pressure in the SHR rats $(193.1 \pm 7.0 \mathrm{mmHg}$, mean \pm SEM) was significantly higher than in the WKY rats $(143.0 \pm 2.6 \mathrm{mmHg}, \mathrm{p}<$ $0.01)$, and heart rate was also greater in the SHR rats $(437 \pm 7 / \mathrm{min})$ than in the WKY rats $(329 \pm 7 / \mathrm{min}, \mathrm{p}<0.01)$.

\section{Stress Experiment 2}

Twelve of the 25 male WKY rats maintained as described above were exposed to 'RESTRICTED SPACE' stress from $9: 30$ to $13: 30$, and the other 13 male WKY rats were not. Urine samples were collected from the untreated rats from $9: 30$ to $13: 30$ on the stress experiment day and the preceding control day.

\section{Urine Analysis}

Each urine sample was diluted to $6 \mathrm{ml}$ with distilled water and stored at -20 ${ }^{\circ} \mathrm{C}$ until analysis of adrenaline, noradrenaline, dopamine ${ }^{9)}$, and corticosterone ${ }^{8)}$ was performed. Creatinine in urine was also measured by the method described by Technicon Instrument Co. ${ }^{11)}$ Excretory rates of catecholamines and corticosterone are expressed as $\mathrm{pmol}$ or $\mathrm{nmol} / \mathrm{mg}$ creatinine.

\section{Statistical Analysis}

Statistical significance was tested by the paired t-test or two-tailed t-test, and differences of $\mathbf{P}<0.05$ were considered significant. To evaluate the effect of 
stress, the hormone values in the stress period and the recovery period were compared with those for the corresponding time period on the preceding control day.

\section{RESULTS}

\section{(1) Stress Experiment 1}

Urinary excretion of corticosterone, adrenaline, noradrenaline and dopamine by SHR and WKY rats exposed to various stresses in stress experiment 1 are shown in Table 1. The values during the recovery period are given in Table 2.

\section{IMMOBILIZATION}

Two-hour immobilization induced significant increases in the urinary excretion of corticosterone, adrenaline and noradrenaline in WKY rats. The percentage increases compared to the control value were $125 \%$ for corticosterone, $121 \%$ for adrenaline and $31 \%$ for noradrenaline. The adrenaline level remained elevated during the recovery period. Dopamine, on the other hand, decreased significantly by $15 \%$ during the 2 -hour immobilization experiment. Similar results were obtained in the 20-min immobilization experiment, although the increments were less pronounced. In the SHR rats, the urinary output of corticosterone, adrenaline and noradrenaline increased by $192 \%, 268 \%$ and $62 \%$, respectively, and dopamine output tended to decrease (14\%) during the 2-hour immobilization. The adrenaline and corticosterone values durig the 2-hour immobilization were significantly higher in the SHR rats than in the WKY rats.

\section{'RESTRICTED SPACE' CONDITION}

Reduction of free space by placing a plastic box in the rat cage produced a significant increase in adrenaline and corticosterone excretion and a decrease in dopamine excretion in the WKY rats. In the SHR rats, only an increase in adrenaline was observed under these conditions. In the recovery period, hardly any difference in hormone levels was found between the stress-treated day and the preceding control day in either SHR or WKY rats.

\section{'NEW RAT-IN-ROOM' CONDITION}

When 3 new SHR rats were introduced into the experiment room, WKY rats showed a significant increase in adrenaline excretion, as compared to the control level in the preceeding 'RESTRICTED SPACE' experiment. In the recovery period, the adrenaline returned to control levels. In the SHR rats, no significant changes were observed as a result of this form of stress. 
Table 1. Urinary excretion of corticosterone, adrenaline, noradrenaline and dopamine in WKY and SHR rats exposed to various stresses.

\begin{tabular}{|c|c|c|c|c|}
\hline & Corticosterone & Adrenaline & Noradrenaline & Dopamine \\
\hline \multicolumn{5}{|c|}{ RESTRICTED SPACE ( 2 hours) } \\
\hline WKY Control day & $30.5 \pm 2.1$ & $51.9 \pm 3.8$ & $671 \pm 35$ & 4. $94 \pm 0.24$ \\
\hline Stress day & $61.9 \pm 11.7^{*}$ & $111.7 \pm 15.6^{* *}$ & $727 \pm 52$ & 4. $52 \pm 0.16^{*}$ \\
\hline SHR Control day & $30.0 \pm 9.5$ & $60.3 \pm 5.3$ & $674 \pm 35$ & $7.15 \pm 0.36^{\dagger \dagger}$ \\
\hline Stress day & 34. $0 \pm 3.3^{\dagger}$ & 106. $4 \pm 8.0^{* *}$ & $678 \pm 32$ & $6.23 \pm 0.29^{\dagger \dagger}$ \\
\hline \multicolumn{5}{|c|}{ NEW RAT IN ROOM (4 hours) } \\
\hline WKY Stress day ${ }^{a}$ & 33. $8 \pm 1.8$ & $81.4 \pm 6.6^{* *}$ & $717 \pm 46$ & 4. $84 \pm 0.24$ \\
\hline \multirow{2}{*}{\multicolumn{2}{|c|}{$\begin{array}{l}\text { SHR Stress day }{ }^{a} \quad 25.2 \pm 2.4^{\prime} \\
\text { IMMOBILIZATION (20 } \mathrm{min})\end{array}$}} & $72.0 \pm 6.9$ & $626 \pm 23$ & $6.63 \pm 0.37^{\dagger \dagger}$ \\
\hline & $\min )$ & & & \\
\hline WKY Control day & $29.8 \pm 1.9$ & $69.8 \pm 4.0$ & $60.3 \pm 17$ & 3. $80 \pm 0.20$ \\
\hline Stress day & 46. $1 \pm 4.5^{* *}$ & 123. $9 \pm 11.2^{* *}$ & $730 \pm 24^{* *}$ & $3.66 \pm 0.21$ \\
\hline SHR Control day & $34.9 \pm 2.3$ & 54. $5 \pm 5.7^{\dagger}$ & $600 \pm 16$ & $5.95 \pm 0.32^{\dagger \dagger}$ \\
\hline Stress day & $60.6 \pm 6.7$ & 144. $2 \pm 40.4$ & $788 \pm 86$ & $5.13 \pm 0.48^{\dagger}$ \\
\hline \multicolumn{5}{|c|}{ IMMOBILIZATION ( 2 hours) } \\
\hline WKY Control day & 34. $8 \pm 2.9$ & $80.5 \pm 6.4$ & $650 \pm 23$ & $3.74 \pm 0.18$ \\
\hline Stress day & $78.3 \pm 18.3^{*}$ & $178.6 \pm 13.1^{* *}$ & $855 \pm 74^{*}$ & 3. $17 \pm 0.16^{* *}$ \\
\hline SHR Control day & 48. $1 \pm 6.4$ & $73.7 \pm 5.1$ & $636 \pm 28$ & $5.18 \pm 0.32^{t \dagger}$ \\
\hline Stress day & 140. $6 \pm 19.9^{* * \dagger}$ & $271.7 \pm 38.7^{* * \dagger}$ & $1031 \pm 98^{* *}$ & $4.47 \pm 0.28^{\dagger \dagger}$ \\
\hline \multicolumn{5}{|c|}{ NOISE (100 dB(C), 4 hours) } \\
\hline WKY Control day & 37. $1 \pm 4.1$ & $72.8 \pm 5.2$ & $644 \pm 27$ & 4. $57 \pm 0.24$ \\
\hline Stress day & $64.8 \pm 6.4^{* *}$ & $76.4 \pm 5.0$ & $646 \pm 28$ & 3. $92 \pm 0.20$ \\
\hline SHR Control day & $30.5 \pm 1.6$ & $60.9 \pm 3.6$ & $654 \pm 25$ & $6.62 \pm 0.32^{t \dagger}$ \\
\hline Stress day & $63.3 \pm 6.7^{* *}$ & $63.5 \pm 4.7$ & $592 \pm 19^{*}$ & $6.05 \pm 0.23^{\dagger \dagger}$ \\
\hline \multicolumn{5}{|c|}{ NOISE ( $87 \mathrm{~dB}(\mathrm{C}), 4$ hours) } \\
\hline WKY Control day & 34. $8 \pm 2.3$ & $71.6 \pm 4.9$ & $620 \pm 32$ & 4. $29 \pm 0.10$ \\
\hline Stress day & 32. $2 \pm 3.1$ & $49.8 \pm 3.7^{* *}$ & $540 \pm 24^{* *}$ & 3. $82 \pm 0.16^{* *}$ \\
\hline SHR Control day & 36. $8 \pm 1.9$ & $59.8 \pm 5.5$ & $586 \pm 31$ & $6.03 \pm 0.25^{\dagger \dagger}$ \\
\hline Stress day & $33.6 \pm 3.7$ & 41. $8 \pm 5.9^{*}$ & $531 \pm 29$ & $6.26 \pm 0.26^{\dagger \dagger}$ \\
\hline \multicolumn{5}{|c|}{ NOISE ( $75 \mathrm{~dB}(\mathrm{C}), 4$ hours) } \\
\hline WKY Control day & $35.9 \pm 2.6$ & $72.2 \pm 4.2$ & $632 \pm 27$ & 4. $43 \pm 0.16$ \\
\hline Stress day & $39.2 \pm 2.9$ & $58.6 \pm 4.3^{* *}$ & $594 \pm 31^{*}$ & 4. $54 \pm 0.18$ \\
\hline SHR Control day & $33.7 \pm 1.3$ & $60.3 \pm 4.1$ & $620 \pm 23$ & $6.33 \pm 0.27^{\dagger \dagger}$ \\
\hline Stress day & $29.6 \pm 2.6^{\dagger}$ & $56.7 \pm 6.0$ & $561 \pm 32$ & 6. $58 \pm 0.10^{\dagger \dagger}$ \\
\hline
\end{tabular}

SHR rats $(\mathrm{N}=7)$ and WKY rats $(\mathrm{N}=8)$ were exposed to stress at 10:00, and urine samples from each rat were collected between 10:00 and 14:00 in stress-exposure days and on the preceding control days. Values are means \pm SEM of urinary excretion expressed as $\mathrm{pmol} / \mathrm{mg}$ creatinine for adrenaline, noradrenaline and corticosterone, or as $\mathrm{nmol} / \mathrm{mg}$ creatinine for dopamine. ${ }^{*}: \mathrm{p}<0.05,{ }^{* *}: \mathrm{p}<0.01$ for the control period vs. the stress period, and ${ }^{\dagger}: \mathrm{p}<0.05,{ }^{\dagger \dagger}: \mathrm{p}<$ 0.01 for SHR rats vs. WKY rats in the corresponding period. ${ }^{a}$ : the values were compared to the control values of the preceding 'RESTRICTED SPACE' experiment. 
Table 2. Urinary excretion of corticosterone, adrenaline, noradrenaline and dopamine in WKY rats and SHR rats after stress exposure.

\begin{tabular}{|c|c|c|c|c|}
\hline & Corticosterone & Adrenaline & Noradrenaline & Dopamine \\
\hline \multicolumn{5}{|c|}{ RESTRICTED SPACE ( 2 hours) } \\
\hline WKY Control day & $22.7 \pm 1.6$ & $61.6 \pm 5.3$ & $674 \pm 32$ & 4. $81 \pm 0.23$ \\
\hline Stress day & $28.1 \pm 2.5$ & $59.4 \pm 2.9$ & $660 \pm 30$ & 4. $85 \pm 0.24$ \\
\hline SHR Control day & 10. $1 \pm 1.1$ & $45.3 \pm 2.3$ & $681 \pm 20$ & $7.69 \pm 0.32^{\dagger \dagger}$ \\
\hline Stress day & $12.6 \pm 1.7$ & $43.3 \pm 3.1$ & $613 \pm 34$ & $6.42 \pm 0.29^{* t+}$ \\
\hline \multicolumn{5}{|c|}{ NEW RAT IN ROOM ( 4 hours) } \\
\hline WKY Stress day ${ }^{a}$ & $22.1 \pm 1.7$ & $67.6 \pm 5.7$ & $624 \pm 30^{*}$ & 4. $55 \pm 0.18$ \\
\hline SHR Stress day ${ }^{a}$ & $9.9 \pm 1.6^{\dagger \dagger}$ & $42.3 \pm 3.8^{\dagger \dagger}$ & $566 \pm 21^{*}$ & $6.74 \pm 0.35^{\dagger \dagger}$ \\
\hline \multicolumn{5}{|c|}{ IMMOBILIZATION (20 min) } \\
\hline WKY Control day & $23.6 \pm 1.8$ & $88.7 \pm 7.5$ & $544 \pm 17$ & 3. $88 \pm 0.18$ \\
\hline Stress day & 27. $2 \pm 1.8$ & $97.3 \pm 6.4^{*}$ & $616 \pm 16^{* *}$ & 4. $03 \pm 0.24$ \\
\hline SHR Control day & $15.3 \pm 1.3^{\dagger \dagger}$ & $38.9 \pm 2.8^{\dagger \dagger}$ & $498 \pm 18$ & $5.86 \pm 0.25^{\dagger \dagger}$ \\
\hline Stress day & 18. $6 \pm 2.4^{\dagger}$ & 53. $6 \pm 4.0^{* t \dagger}$ & $573 \pm 45$ & $5.63 \pm 0.66^{\dagger}$ \\
\hline \multicolumn{5}{|c|}{ IMMOBILIZATION (2 hours) } \\
\hline WKY Control day & 24. $0 \pm 1.1$ & $85.2 \pm 5.5$ & $572 \pm 17$ & $3.93 \pm 0.17$ \\
\hline Stress day & $24.9 \pm 2.2$ & $100.6 \pm 2.8^{*}$ & $629 \pm 28$ & 4. $05 \pm 0.21$ \\
\hline SHR Control day & $22.8 \pm 2.3$ & 66. $2 \pm 8.1$ & $580 \pm 26$ & $5.60 \pm 0.26^{\dagger t}$ \\
\hline Stress day & 24. $3 \pm 2.0$ & 93. $0 \pm 7.5^{* \dagger}$ & $696 \pm 54^{*}$ & $5.31 \pm 0.23^{\dagger \dagger}$ \\
\hline \multicolumn{5}{|c|}{ NOISE (100 dB(C), 4 hours) } \\
\hline WKY Control day & $27.8 \pm 4.6$ & $73.2 \pm 5.5$ & $590 \pm 25$ & 4. $28 \pm 0.28$ \\
\hline Stress day & 24. $2 \pm 2.9$ & $68.8 \pm 6.7$ & $614 \pm 20$ & 4. $64 \pm 0.18$ \\
\hline SHR Control day & 13. $3 \pm 1.2^{\dagger}$ & $39.9 \pm 3.6^{t t}$ & $584 \pm 23$ & $6.58 \pm 0.42^{\dagger \dagger}$ \\
\hline Stress day & 23. $6 \pm 2.0^{* *}$ & $49.6 \pm 7.8$ & $552 \pm 26$ & $7.02 \pm 0.36^{\dagger t}$ \\
\hline \multicolumn{5}{|c|}{ NOISE (87 dB(C), 4 hours) } \\
\hline WKY Control day & $24.5 \pm 2.3$ & $89.9 \pm 7.1$ & $518 \pm 18$ & 4. $27 \pm 0.14$ \\
\hline Stress day & $24.6 \pm 2.1$ & $71.8 \pm 6.8^{* *}$ & $507 \pm 22$ & 4. $02 \pm 0.30$ \\
\hline SHR Control day & 16. $6 \pm 1.5^{\dagger}$ & $47.5 \pm 4.8^{\dagger \dagger}$ & $504 \pm 23$ & $6.02 \pm 0.26^{\dagger t}$ \\
\hline Stress day & $18.0 \pm 3.1$ & $41.7 \pm 5.2^{\dagger \dagger}$ & $490 \pm 23$ & $6.03 \pm 0.33^{\dagger+}$ \\
\hline \multicolumn{5}{|c|}{ NOISE (75 dB(C), 4hours) } \\
\hline WKY Control day & 26. $2 \pm 2.9$ & $81.5 \pm 3.9$ & $554 \pm 21$ & 4. $27 \pm 0.20$ \\
\hline Stress day & $22.9 \pm 1.3$ & $75.4 \pm 3.8^{*}$ & $582 \pm 25^{*}$ & $4.43 \pm 0.09$ \\
\hline SHR Control day & $14.9 \pm 1.1^{\dagger \dagger}$ & $43.7 \pm 3.0^{t t}$ & $544 \pm 21$ & $6.30 \pm 0.32^{\dagger t}$ \\
\hline Stress day & 13. $2 \pm 2.2^{\dagger \dagger}$ & $45.0 \pm 4.3^{t \dagger}$ & $549 \pm 36$ & $6.64 \pm 0.27^{\dagger \dagger}$ \\
\hline
\end{tabular}

SHR rats $(\mathrm{N}=7)$ and WKY rats $(\mathrm{N}=8)$ were exposed to stress at 10:00, and urine samples from each rat were collected between 14:00 and 18:00 on stress-exposure days and on the preceding control days. For other details, see the footnote to Table 1. 


\section{NOISE EXPOSURE}

Exposure to intermittent $100 \mathrm{~dB}(\mathrm{C})$ noise produced a marked increase in urinary excretion of corticosterone in both WKY and SHR rats. Corticosterone levels were still elevated in the recovery period in the SHR rats, but no difference was observed in the WKY rats. Noradrenaline excretion showed a slight but significant decrease during $100 \mathrm{~dB}(\mathrm{C})$ noise exposure in SHR rats. In the WKY rats, catecholamines did not exhibit any noise-related changes during either the exposure period or the recovery period at this noise level.

Lower noise levels produced no changes in corticosterone excretion but significant decreases in adrenaline, noradrenaline and dopamine excretion in the WKY rats. SHR rats also showed significantly reduced adrenaline output during noise exposure at $87 \mathrm{~dB}(\mathrm{C})$.

\section{Comparison of SHR and WKY rats on control days}

In the no-stress control days, urinary levels of adrenaline, noradrenaline and corticosterone from 14:00 to 18:00 were lower in the SHR rats than in the WKY rats, although hardly any difference was observed during the period from $10: 00$ to $14: 00$. Dopamine excretion was considerably higher in the SHR rats than in the WKY rats during both peirods, with or without stress exposure.

\section{(2) Stress Experiment 2}

The urinary catecholamine and corticosterone output of WKY rats in stress experiment 2 is shown in Table 3. The data indicate that when rats were exposed to 'RESTRICTED SPACE' stress, the roommate rats unexposed to stress displayed a significant increase in adrenaline excretion and a slight decrease in dopamine excretion. No significant changes were observed in noradrenaline or corticosterone excretion in the untreated rats.

Table 3. Urinary excretion of corticosterone, adrenaline, noradrenaline and dopamine in WKY rats exposed to psychological stress.

\begin{tabular}{lllcl}
\hline & Corticosterone & \multicolumn{1}{c}{ Adrenaline } & Noradrenaline & \multicolumn{1}{c}{ Dopamine } \\
\hline Control day & $44.7 \pm 5.1$ & $71.7 \pm 6.7$ & $567 \pm 17$ & $5.25 \pm 0.17$ \\
Stress day & $43.0 \pm 4.5$ & $85.3 \pm 6.3^{* *}$ & $585 \pm 15$ & $5.00 \pm 0.18^{*}$ \\
\hline
\end{tabular}

Urine samples from 13 WKY rats maintained under 12-hour light $(20: 30-8: 30)$ and 12-hour dark $(8: 30-20: 30)$ conditions were collected between $9: 30$ and $13: 30$ while other 12 WKY rats in the same room were treated with 'restricted space' stress. In the preceding control day, urine collection was performed in the same manner. For additional details, see the footnote to Table 1. 


\section{Discussion}

The present study demonstrated that immobilization for 2 hours causes significant increases in the urinary excretion of corticosterone, adrenaline and noradrenaline in rats. Immobilization-induced increases in urinary adrenaline and noradrenaline in rats have also been reported by Kvetnansky et $\mathrm{al}^{12)}$. Elevations of plasma levels of adrenaline, noradrenaline and corticosterone have been demonstrated in rats exposed to immobilization ${ }^{1)}$ and footshock ${ }^{2)}$. The results indicate that these severe stresses induce enhanced sympathetic-adrenomedullary system and pituitary-adrenocortical axis activity.

Urinary excretion of dopamine significantly decreased in the immobilizationexposed rats in the present experiment. Our previous data ${ }^{13)}$ indicated that footshock also induces a decrease in dopamine excretion in the rat. These results are consistent with the findings of Mizutani et $a l^{14)}$ who stated that repeated immobilization provokes a significant decrease in the urinary output of free dopamine in the rat. It might be concluded that immobilization and footshock cause a decrease in the urinary excretion of free dopamine in these animals. Swimming stress, however, seems to increase urinary dopamine levels, as well as adrenaline, noradrenaline and corticosterone levels, in the rat. ${ }^{13)}$ The reason for the different responses of urinary dopamine to immobilization and swimming is not clear.

Several workers have investigated the effect of relatively mild stress on the pituitary-adrenal and sympathetic-adrenal systems. Popper et al. ${ }^{7)}$ and Buler et $\left.a l .{ }^{6}\right)$ reported that handling induces increased plasma levels of adrenaline, norad renaline and corticosterone in the rat. Elevated plasma levels of these hormones were also observed when rats were placed in a novel cage or when animals was exposed to psychological stress ${ }^{5}$. Noise exposure for several minutes was shown to increase plasma levels of adrenaline, noradrenaline and corticosterone in the rat $^{15)}$. However, the response of urinary catecholamine and corticosterone levels to mild stress is not fully understood.

The present study was carried out to clarify the effects of mild stress on the urinary excretion of adrenaline, noradrenaline, dopamine and corticosterone in the rat. When an unfamiliar plastic box was placed in the rat cage, considerably reducing free space, urinary excretion of adrenaline and corticosterone in the rat increased, with no significant change in noradrenaline excretion. When new rats were introduced into the experiment room, an increase in urinary adrenaline were observed, but no change in noradrenaline or corticosterone excretion was detected in the senior rats. This is consistent with the results of stress experiment 2 in the present study, which showed an increase in adrenaline in rats when other rats in the same experiment room were exposed to stress. Exposure to $100 \mathrm{~dB}(\mathrm{C})$ noise induced only an increase in corticosteroe in the rats, and lower levels of noise 
reduced adrenaline, noradrenaline and dopamine levels, with no change in corticosterone excretion. These results suggest that severe stress like immobilization causes a marked change in all 4 hormones, and that relatively mild stress produces a less pronounced but nevertheless significant change in 1 to 3 , but not all, hormones in the rat. Stress-induced changes in hormone excretion were still observed 4 hours following exposure to severe stress, but hardly detected at all in the case of mild stress, indicating more rapid recovery from mild stress than from severe stress.

The pituitary-adrenal and symathetic-adrenal systems have long been believed to be involved in stress reactions. As described above, severe stress has a great influence on both systems. In the present study, however, $100 \mathrm{~dB}(\mathrm{C})$ noise exposure increased only pituitary-adrenal activity, and a new or strange environment induced significant and marked changes only in adreno-medullary hormones, i. e., catecholamine and corticosterone responses were dissociated in these mild stresses in the rat, suggesting different physiological roles of the two systems in stress reactions. According to Lundberg and Frankenhaeuser ${ }^{16)}$, the responses of the two systems correspond to different psychological states in man. They thought that pituitary-adrenal activation was associated with the negative feeling of distress, and that sympatho-adrenal activation was related to feelings of alertness and action proneness. This concept is helpful in evaluating the different responses of catecholamines and corticosterone to mild stress.

McCarty and Kopin ${ }^{10)}$ demonstrated that SHR rats exhibit a greater response of plasma adrenaline and noradrenaline levels to footshock than WKY rats. Kvetnansky et al. ${ }^{17)}$ also reported that during immobilization SHR rats had significantly higher circulating levels of adrenaline, noradrenaline and corticosterone than WKY rats. We confirmed by urine analysis that immobilizationinduced increases in adrenaline, noradrenaline and corticosterone are greater in SHR rats than in WKY rats. The greater responsiveness of the sympatheticadrenal system to stress in SHR rats has been thought to contribute to the development of hypertension ${ }^{18)}$. However, according to the present results, mild stress causes less pronounced changes in SHR rats than in WKY rats, judging from the urinary excretion of catecholamines and corticosterone. Furthermore, in the urine collected between $14: 00$ and 18:00 on the no-stress control days, the adrenaline and corticosterone values were significantly lower in the SHR rats than in the WKY rats. It appears that SHR rats become hypertensive at the age of 8 weeks, at the latest, even if the rat is maintained under ordinary, less-stressful conditions. The present findings do not support the hypothesis that stressinduced hyperactivity of the sympathetic-adrenomedullary system promotes the development of hypertension in SHR rats $^{18)}$, at least under ordinary, unstressed laboratory conditions.

On the other hand, urinary excretion of dopamine by SHR rats was consistently higher than that of WKY rats. The data presented by Gordon et al. ${ }^{19)}$ 
could be interpreted as indicating that urinary dopamine output is more elevated in hypertensive subjects than in normotensive subjects. Yoshimura et al. $^{20)}$ suggested in their experimental study of hypertensive rats that dopamine participates in the development of hypertension, because the augmentation of dopaminergic activity by administration of bromocropitine, a dopamine agonist, suppressed the increase of blood pressure in DOCA-salt hypertensive rats, while carbipoda, an inhibitor of dopa decarboxylase, accelerated the development of hypertension in SHR rats. It appears that dopamine participates, at least partially, in the mechanisms of development and maintenance of hypertension in SHR rats.

\section{REFERENCES}

1) Kvetnansky R, Weise VK, Thoa NB, Kopin IJ. Effects of chronic guanethidine treatment and adrenal medullectomy on plasma levels of catecholamines and corticosterone in forcibly immobilized rats. J. Pharmacol. Exp. Ther. 1979; 209: 287-91.

2) Natelson BH, Creighton D, McCarty R, Tapp WN, Pitman D, Ottenweller JE. Adrenal hormonal indices of stress in laboratory rats. Physiol. Behav. 1987; 39: 117-25.

3) Baldwin DM, Colombo JA, Sawyer CH. Plasma prolactin, LH, and corticosterone in rats exposed to a novel environment. Am. J. Physiol. 1974; 226: 1366-9.

4) Dohler KD, Gartner K, Muhlen A von zur, Dohler U. Activation of anterior pituitary, thyroid and adrenal gland in rats after disturbance stress. Acta Endocrinol. 1977; 86: 489-97.

5) Fokkema DS, Smith K, Gugten J van der, Koolhaas JM. A coherent pattern among social behavior, blood pressure, corticosterone and catecholamine measures in individual male rats. Physiol. Behav. 1988; 42: 485-9.

6) Buler HU, Da Prada M, Haefely W, Picotti GB. Plasma adrenaline, noradrenaline and dopamine in man and different animal species. J. Physiol. 1978; 276: 311-20.

7) Popper CW, Chiueh CC, Kopin IJ. Plasma catecholamine concentrations in unanaethetized rats during sleep, wakefulness, immobilization and after decapitation. J. Pharm. Exp. Ther. 1977; 202: 144-8.

8) Sudo A. Analysis of corticosterone in rat urine by high-performance liquid chromatography and fluorimetry using post-column reaction with sulphuric acid. J. Chromatogr. 1990; 528: 453-8.

9) Sudo A. Measurement of adrenaline in the rats brain by high-performance liquid chromatography with fluorometric detection. Ind. Health, 1982; $20: 151-6$.

10) McCarty R, Kopin IJ. Alterations in plasma catecholamines and behavior during acute stress in spontaneously hypertensive and Wistar-Kyoto normotensive rats. Life Sci. 1978; 22: 997-1006.

11) Technicon Instrument Corporation. Technicon AutoAnalyzer Methodology, N-11a (creatinine). Chauncy, New York, 1963.

12) Kvetnansky R, Mikulaj L. Adrenal and urinary catecholamines in rats during adaptation to repeated immobilization stress. Endocrinol. 1970; 87: 738-43.

13) Unpublished data of Sudo A.

14) Mizutani R, Nakano K. Effect of Vitamin A depletion on stress-induced changes in urinary output of catecholamines. J. Nutr. 1982; 112: 2205-11.

15) De Boer SF, Slangen JL, van der Gugten J. Adaptation of plasma catecholamines and corticosterone responses to short-term repeated noise stress in rats. Physiol. Behav. 1988; 44 : 273-80.

16) Lundberg U, Frankenhaeuser M. Pituitary-adrenal and sympathetic-adrenal correlates of distress and effort. J. Psychosom. Res. 1980; 24: 125-30.

17) Kvetnansky R, McCarty R, Thoa NB, Lake CR, Kopin IJ. Sympathoadrenal responses of spontaneously hypertensive rats to immobilization stress. Am. J. Physiol. 1979; 236: H457-62. 
18) Reid JL, Rubin PC. Catecholamines and blood pressure. In: Trendelenburg U, Weiner N, eds. Catecholamines II ed. Berlin: Springer-Verlag, 1989; 319-56.

19) Gordon MS, Stenkel CA, Colin PR, Hollenberg NK, Williams GH. The role of dopamine in nonmodulating hypertension. J. Clin. Endocrinol. Metab. 1989; 69: 426-32.

20) Yoshimura M, Kambara S, Okabayashi H, Ikegaiki I, Matsuzawa M, Suga K, Takahashi H, Ijichi H. Pathophysiological role of dopamine on the development of hypertension in rats. Jpn. Cir. J. $1987 ; 51: 1226-31$. 